\title{
Observation of Lithium fluorescence X-ray utilizing Superconducting-Tunnel- Junction Array X-ray detector toward in situ mapping analyses of precipitated metal lithium in sold electrolytes of Li-ion batteries.
}

Masahiro Ukibe ${ }^{1}$, Go Fujii ${ }^{1}$, Shigetomo Shiki ${ }^{1}$, and Masataka Ohkubo ${ }^{1}$

1. Nanoelectronics Research Institute, National Institute of Advanced Industrial Science and Technology (AIST), 1-1-1, Umezono, Tsukuba, Ibaraki, Japan

Conditions of lithium in electrodes and an electrolyte of all solid Li-ion batteries, which have been developed as next generation batteries, have a big influence on its' capability as a battery, life time and safety. Usually in those batterie elements, lithium exists as many kinds of compounds or a metal and have many different forms, of which size are ranged from nanometer to micro meters. Thus, to improve those performance, it is very important to evaluate in situ an aging variation of the state of lithium by the battery operation quantitively as well as qualitatively, which includes the spatial distribution of lithium and other composition elements in there.

An EDS analyser combined with a SEM is suitable to obtain spatial and quantitative information on the elemental composition of a sample non-destructively. However, for realizing the analysis of the lithium by SEM-EDS systems, two requirements should be satisfied. First, in order to perform the nanometer scale elemental mapping, it is necessary to operate a SEM at lower accelerating voltage $\left(\mathrm{V}_{\text {acc }}\right)$ less than 1 $\mathrm{keV}$, because electron ranges and interaction volumes in samples become significantly small ( $<$ several $10 \mathrm{~nm})$ at $\mathrm{V}_{\text {acc }}$ of $1 \mathrm{keV}$.[1,2] Secondary, it is necessary to use high performance soft X-ray detectors, which can exhibit high sensitivity and high energy resolution simultaneously, because the fluorescence $\mathrm{X}$-ray analysis of lithium is fairly difficult by silicon drift detectors (SDDs) or Si(Li) detectors used in the conventional EDS analysers due to a low energy ( $\sim 54 \mathrm{eV})$ and a small emission yields $(0.0003[3])$ of Li-K $\alpha$.

In contrast, energy-dispersive X-ray detectors based on arrays of superconducting-tunnel-junctions (STJs) have simultaneously exhibited excellent energy resolution of $<10 \mathrm{eV}$, relatively large detection area of $>1 \mathrm{~mm}^{2}$, high counting rate capability of $>500 \mathrm{kcps}$, and high sensitivity for soft X-rays less than $1 \mathrm{keV}[4,5]$. We have been developing a SEM-EDS analyser utilizing an STJ array (SC-SEM), in order to realize analysis of light elements in advance functional materials with nanometer scale [6].

In this work, as a first step, we performed fluorescence X-ray analysis of an Al plate, and lithium compounds to evaluate the analytical capabilities of SC-SEM for lithium metal at low-accelerationvoltage.

Figure 1 shows a cross-sectional schematic illustration of the SC-SEM. The SC-SEM consisted of an FE-SEM and the STJ array detector. The STJ array was cooled to $0.31 \mathrm{~K}$ on a cold stage of a cryogenfree helium-3 cryostat. X-rays emitted from the sample by the electron beam were detected by the STJ array via the polycapillary collimating X-ray lens and two X-ray windows. In order to improve the collection efficiency, a polycapillary collimating X-ray lens was installed in the system. The overall collection efficiency of the SC-SEM, which includes two X-ray windows, was about $1 \mathrm{mSr}$, which was about 1/100 times smaller than that of the SDDs [6].

Figure 2(a) and (b) shows a fluorescence X-ray peaks of $\mathrm{Al}-\mathrm{L} \alpha$ of $\mathrm{Al}$ plate and $\mathrm{Li}-\mathrm{K} \alpha$ of $\mathrm{LiF}$, respectively. The value of $\mathrm{V}_{\text {acc }}$ and probe current of the SEM were $1 \mathrm{kV}$ and about $2 \mathrm{nA}$, respectively. As you can see, clear peaks of Al- $L \alpha(73 \mathrm{eV})$ and $\mathrm{Li}-K \alpha$ were obtained by SC-SEM. A full width at half-maximum value for Al- $L \alpha$ and Li-K$\alpha$ was about $9 \mathrm{eV}$ and $8 \mathrm{eV}$, respectively. It is found that SC-

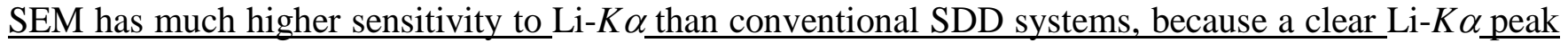


couldn't be obtained by conventional SDD systems at the same $\mathrm{V}_{\text {acc }}$ of $1 \mathrm{keV[7]}$. Those results indicate that SC-SEM may perform the in situ analysis of the precipitated metal lithium in sold electrolytes of all solid Li-ion batteries.

[1] J R Michael, D C Joy and B J Griffin, Microsc. Microanal. 15 (Suppl. S2) (2009) 660.

[2] R Wuhrer and K Moran, IOP Conf. Ser.: Mater. Sci. Eng. 109 (2016) 012019.

[3] Richard B. Firestone. Table of Isotopes, 8th edition, volume 2. Edited by Virginia S. Shirley, with assistant editors Coral M. Baglin, S. Y. Frank Chu, and Jean Zipkin. New York: John Wiley \& Sons, Inc., 1996.

[4] S. Friedrich, J. Harris, W. K. Warburton, M. H. Carpenter, J. A. Hall, and R. Cantor, J. Low. Temp. Phys. 176 (2014) 553.

[5] M. Ukibe, G. Fujii, S. Shiki, Y. Kitajima, and M. Ohkubo, J. Low. Temp. Phys. 184 (2016) 200.

[6] G. Fujii, M. Ukibe, S. Shiki, and M. Ohkubo, X-ray spec., 46 (2017) 325.

[7] O. Hovington, V. Timoshevskii, S. Burgness, H. Demers, P. Statham, R. Gauvin, and K. Zaghib, Scan. 38,(2016)571.

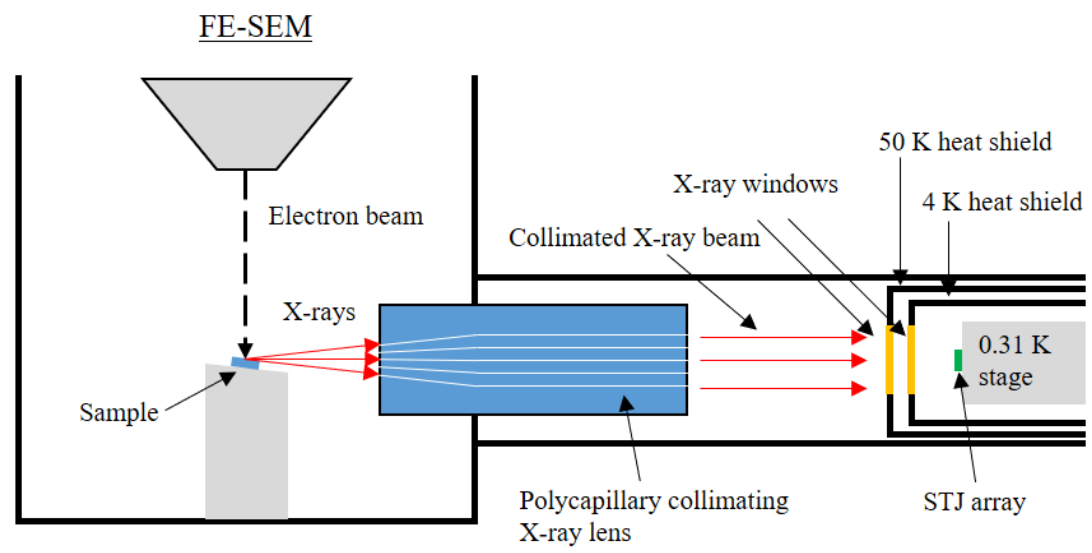

Figure 1. Cross-sectional schematic illustration of the SC-SEM
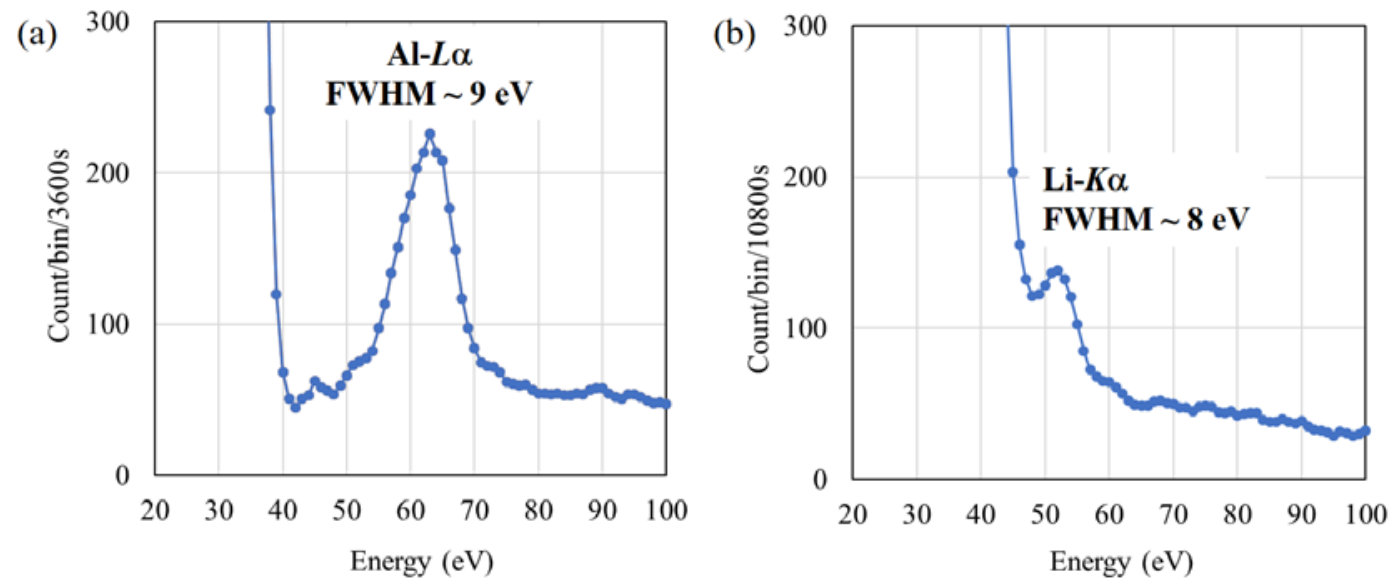

Figure 2. Fluorescence X-ray peaks obtained by SC-SEM at $\mathrm{V}_{\text {acc }}$ of $1 \mathrm{keV}$ (a) Al-L $\alpha$ and (b) Li-K $\alpha$. 